\title{
On One Connexion Between Levi-Civita Parallelism and Einstein's Teleparallelism.
}

\author{
By R. N. Sen, University of Dacca. \\ (Received in revised form 6th January, 1931. \\ Read 7th November 1930.)
}

\$1. In a recent paper ${ }^{1}$ Einstein has proposed a new type of geometry in which it is possible to give an arbitrary vector in the field a displacement which conserves parallelism at a distance. It may be briefly stated as follows:

Let the metric of the space, supposed to be of $n$ dimensions, be given by

$$
d s^{2}=\sum_{p q} g_{p q} d x_{p} d x_{q}
$$

At each point of the space we consider a set of $n$ unit vectors, denoted by the $n^{2}$ quantities ${ }^{p} h_{q}$, which form an orthogonal ennuple such that

$$
g_{p q_{i}}=\sum_{t} h_{p} h^{t} h_{q}
$$

Now, if at any point we take a direction $V$ and give it a displacement according to the law

$$
\begin{aligned}
& d V^{l}+\sum_{p q} \Gamma_{q p}^{l} V^{p} d x_{q_{1}}=0, \quad(l=1,2, \ldots, n) \\
& \Gamma_{p q}^{l}=\sum_{t} h^{l} \frac{\partial^{t} h_{p}}{\partial x_{q}},
\end{aligned}
$$

where

and ${ }_{p} h^{q}$ is the cofactor of ${ }^{n} h_{q}$ in the determinant $\left|{ }^{p} h_{q}\right|$ divided by this determinant, then the direction finally arrived at, called the parallel to the original one, is unique, i.e., independent of any path along which the transport is carried out. This is Einstein's teleparallelism.

With the parallel transport defined by the equations (1.1) we may associate another by interchanging the indices of $V$ and $d x$ on the right without interchanging those of $\Gamma$, namely

$$
d V^{l}+\sum_{p q} \Gamma_{p q}^{l} V^{q} d x_{p}=0, \quad(l=1,2, \ldots, n) .
$$

These two parallelisms have one important property in common, namely that the auto-parallels are the same in both cases; this is

1 Berl. Sitzungsber (1929), 2. 
seen by putting $d x_{r /} d s$ for $V^{r}$. If the $\Gamma^{\prime}$ s are symmetric in the two lower indic s, so that the two parallelisms are the same, then the space is euclidean. ${ }^{1}$ If we put

$$
\Lambda_{p q}^{l}=\Gamma_{p q}^{l}-\Gamma_{q p}^{l},
$$

then $\Lambda_{p^{\prime}}^{l}$ is a tensor skew in the two lower indices.

Since for any defined type of parallel transport we get a particular form of covariant differentiation, we may reasonably, and without ambiguity, suppose that the covariant derivative of a tensor with respect to (1.2) is obtained by interchanging throughout the two lower indices of $\Gamma$ 's in the same covariant derivative with respect to the teleparallelism (1.1). We shall hereafter refer to (1.2) as the equations for the associated parallel transport.

$\S 2$. By composition with the $h$ 's we obtain from every tensor $A_{\nu . . \quad p}^{\lambda \ldots \mu}$ of any rank ennuplet tensors of lower ranks and an ennuplet scalar which we define in the following way: ${ }^{2}$

For the sake of brevity we consider a tensor $A_{\nu}^{\lambda \mu}$ of rank 3. First of all we set up places by dots on the left side of the tensor corresponding to the positions of the indices on the right and write $\because A_{\nu \mu}^{\lambda \mu}$. We then obtain, for example, ennuplet tensors of ranks 2 and 1 whose components are ennuplet scalar

$$
{ }^{a} \cdot A_{\nu}^{\lambda .}=\sum_{\mu} A_{\nu}^{\lambda \mu}{ }^{a} h_{\mu} \text { and }{ }_{b}^{a} A^{\mu}=\sum_{\lambda_{r}} A^{\lambda \mu}{ }^{a} h_{\lambda b} h^{\nu} \text { respectively, and the }
$$

$$
{ }_{c}^{a b} A=\sum_{\lambda \mu \nu} A_{\nu}^{\lambda \mu}{ }^{b} h_{\lambda}{ }^{a} h_{\mu}{ }_{c} h^{\nu} .
$$

The indices $a, b, c$ are placed on the left of $A$ corresponding to those on the right of $A$ which have been taken away by composition with the $h$ 's.

Conversely, multiplying by ${ }_{a} h^{\mu}$ and summing for $a$, we get

$$
A_{\nu}^{\lambda \mu}=\sum_{a}^{a} \cdot A_{\nu}^{\lambda .} h^{\mu} \text {; }
$$

similarly for others.

An ennuplet scalar possesses the property that if the original tensor is skew in two or more indices, then the corresponding scalar is also skew in the corresponding indices on the left. The same thing is also true of ennuplet tensors which have those indices on the left.

1 R. Zaycoff, Z.'. für Phys., 53 (1929), 719.

:Suggested by Levi-Civita's paper on Einstein's Unified field theory, Berl. Sitzungsb. (1929), 137. 
Let us now consider the ennuplet scalar ${ }_{r q}^{p} \Lambda$ which is derived from the tensor $\Lambda_{\nu \sigma}^{a}$ (defined in (1.3)), namely

$$
{ }_{r q}^{p} \Lambda=\sum_{a \nu \sigma} \Lambda_{\nu \sigma}^{a}{ }^{p} h_{a q} h^{v}{ }_{r} h^{\sigma} .
$$

Ricci constructed a set of important quantities $\gamma_{p q r}$, known as Ricci's coefficients of rotation If we proceed on exactly the same line as is adopted by Levi-Civita in defining $\gamma_{p q},{ }^{1}$ using the associated parallelism instead of Levi-Civita parallelism, then we shall get the quantities ${ }_{:-h}^{p} \Lambda$. We may therefore call the quantities ${ }_{r q}^{p} \Lambda$ the coefficients of rotation with respect to the associated parallel transport. Since these quantities are skew in the two lower indices, we have

$$
\underset{r q}{p} \Lambda+\underset{q r}{p} \Lambda=0, \quad{ }_{q q}^{p} \Lambda=0 ;
$$

the number of these quantities which are independent is, therefore, $\frac{1}{2} n^{2}(n-1)$.

§3. We now proceed to find the connexion between $\underset{r q}{p} \Lambda$ and $\gamma_{p q r}$. We have

$$
\gamma_{p q r}=\sum_{i j}\left({ }^{p} h_{i}\right)_{j q} h^{i}{ }_{r} h^{j}
$$

where ()$_{j}$ is used to denote covariant differentiation with respect to Levi-Civita parallelism.

But

$$
\begin{aligned}
\left.\sum_{p} h_{p} h^{p} h_{i}\right)_{j} & =\sum_{p}{ }_{p} h^{k} \frac{\partial^{p} h_{i}}{\partial x_{j}}-\sum_{t p}\left\{\begin{array}{c}
t \\
i j
\end{array}\right\}{ }^{p} h_{t p} h^{k} \\
& =\Gamma_{i j}^{\kappa}-\left\{\begin{array}{c}
k \\
i j
\end{array}\right\} .
\end{aligned}
$$

Denote $\sum_{p} h^{k}\left({ }^{p} h_{i}\right)_{j}$ by $\mathbf{T}_{i j}^{k}$. So

$$
\begin{aligned}
\Gamma_{i j}^{k} & =\left\{\begin{array}{l}
k \\
i j
\end{array}\right\}+\mathbf{T}_{i j}^{k}, \text { and } \\
\gamma_{p q r} & =\sum_{k i j} \mathbf{T}_{i j}^{k}{ }^{k} h_{k}{ }_{q^{i}} h^{i}{ }_{r} h^{j} .
\end{aligned}
$$

Again, since the covariant derivatives of $g_{p q}$ with respect to the teleparallelism vanish, we have

So

$$
\frac{\partial g_{p q}}{\partial x_{r}}=\Sigma_{t}\left(g_{t p} \Gamma_{q r}^{t}+g_{t q} \Gamma_{p r}^{t}\right)
$$

$$
2[i j, l]=\sum_{t}\left\{g_{t i} \Lambda_{l j}^{t}+g_{t j} \Lambda_{l i}^{t}+g_{t l}\left(2 \Gamma_{i j}^{t}-\Lambda_{i j}^{t}\right)\right\},
$$

where $[i j, l]$ is Christoffel symbol of the first kind.

1 Absolute Differential Calculus (1927), 268. 
Therefore

$$
2\left\{\begin{array}{c}
k \\
i j
\end{array}\right\}=2 \Gamma_{i j}^{k}-\left\{\Lambda_{i j}^{k}+\sum_{t l}^{\Sigma} g^{l k}\left(g_{t i} \Lambda_{j l}^{t}+g_{t j} \Lambda_{i l}^{t}\right)\right\} .
$$

Comparing this with (3.1), we get

$$
2 T_{i j}^{k}=\Lambda_{i j}^{k}+\sum_{t l} g^{l k}\left(g_{t i} \Lambda_{j l}^{t}+g_{t j} \Lambda_{i l}^{t}\right)
$$

Now substitute for the $g$ 's their values in terms of the $h$ 's, namely

$$
g_{t i}=\sum_{a}^{a} h_{t}{ }^{a} h_{i}, \quad g^{l k}=\sum_{b} h_{b}{ }_{b} h^{k},
$$

and perform the multiplication necessary to form the ennuplet tensors. We shall have

$$
2 T_{i j}^{k}=\Lambda_{i j}^{k}+\sum_{a b} h^{k}\left({ }^{a} h_{i b}{ }^{a} \Lambda_{j}+{ }^{a} h_{j b}{ }^{a} \Lambda_{i}\right)
$$

Finally, multiplying by ${ }^{p} h_{k q} h^{i}{ }_{r} h^{j}$ and summing for $i, j, k$,

$$
2 \sum_{i j k} T_{i j}^{k}{ }^{p} h_{k q} h^{i}{ }_{r} h^{j}={ }_{r q}^{\prime \prime} \Lambda+\underset{p r}{q} \Lambda+\underset{p q}{r} \Lambda \text {. }
$$

Hence comparing (3.2) with (3.3), we have the interesting relation

$$
2 \gamma_{p q r}={ }_{r q}^{\nu} \Lambda+\underset{p r}{q} \Lambda+{ }_{p q}^{r} \Lambda \text {. }
$$

We thus see that there is a simple connexion between Ricci's coefficients of rotation and the coefficients of rotation with respect to the associated parallel transport. The quantities $\gamma_{p q r}$ are known to be skew in the first two indices; this may also be seen from the fact that the quantities ${ }_{r q}^{\mu} \Lambda$ are skew in the two lower indices. , e therefore have

$$
\begin{aligned}
& \underset{r q}{p} \Lambda=\gamma_{p q r}-\gamma_{p r q}, \quad \underset{p q}{p} \Lambda=\gamma_{p q p}, \\
& { }_{p r}^{q} \Lambda+{ }_{p q}^{r} \Lambda=\gamma_{p q r}+\gamma_{p r q} .
\end{aligned}
$$

The geometrical significance of (3.4) may be stated thus:

If for an infinitesimal displacement of a point $P$, carrying two Jines ${ }^{p} h, q h$ in such $a$ way that these lines are carried by the local and the associated parallel transports respectively, along a third line ${ }^{r} h$ of the orthogonal ennuple at $P$, the resulting change in the cosine of the angle between ${ }^{p} h, q h$ be denoted by $\frac{\partial \cos \Theta_{p(q)}}{\partial s_{r}} d s_{r}$, and if for a similar kind of displacement of the same point with Levi-Civita parallel transport, the resulting change in the cosine of the same angle be denoted by $\frac{\partial \cos \Theta_{p[q]}}{\partial s_{r}} d s$, then these changes are related by

$$
2 \frac{\partial \cos \Theta_{p[q]}}{\partial s_{r}}=\frac{\partial \cos \Theta_{p(q)}}{\partial s_{r}}+\frac{\partial \cos \Theta_{q(r)}}{\partial s_{p}}+\frac{\partial \cos \Theta_{r(q)}}{\partial s_{p}} .
$$

